\title{
REMARKS ON A PAPER OF D. LUDWIG
}

\author{
BY V. GUILLEMIN AND D. SCHAEFFER ${ }^{1}$ \\ Communicated by I. M. Singer, September 26, 1972
}

We consider the reduced wave equation

$$
\Delta u+k^{2} u=0
$$

with initial data prescribed on a hypersurface $Z \subset R^{n}$. It is well known that for points near $Z$, (A) has an asymptotic solution of the form:

$$
a_{+}(x, k) e^{i k \phi_{+}}+a_{-}(x, k) e^{i k \phi_{-}}
$$

where $a_{ \pm}(x, k)$ is an asymptotic series in $k^{-1}$ and $\phi_{ \pm}$is a solution of the eikonal equation: $(\nabla \phi)^{2}=1$ with initial data $\phi_{ \pm}=0$ on $Z$. The nonlinear initial value problem defining $\phi_{ \pm}$may be solved locally by ray tracing, but in general these rays have an envelope (often called a caustic), so a global solution does not exist. This limits the domain in which an asymptotic series of the form (A) can be valid. In [4] Ludwig has derived an expansion valid near a caustic which involves the Airy function. In this note we show how Ludwig's result follows naturally from the combination of two well established mathematical disciplines: Maslov's global theory of asymptotics $([5],[6])$ and the theory of singularities of differentiable mappings. In particular, the application of singularity theory leads to an asymptotic expansion in three dimensions at an intersection of the two focal surfaces, a question left unanswered by Ludwig.

The central idea in the Maslov theory is to consider multiple valued solutions of the eikonal equation. This is most elegantly done with the formalism of Lagrangian manifolds. (See for example [3].) If $X$ is a smooth manifold of dimension $n$, a Lagrangian manifold is an $n$-dimensional submanifold of the cotangent bundle $T^{*}(X)$ on which the symplectic 2-form $\sum d x_{i} \wedge d \xi_{i}$ vanishes. For any function $\phi \in C^{\infty}(M)$, the graph of $d \phi$,

$$
\Gamma(d \phi)=\left\{\left(x, d \phi_{x}\right): x \in X\right\},
$$

is a Lagrangian manifold $\Lambda$ such that the natural projection $\pi: T^{*}(X) \rightarrow X$, restricted to $\Lambda$, is a diffeomorphism. Conversely, if $\Lambda$ is a Lagrangian manifold and if $\pi: \Lambda \rightarrow X$ is a diffeomorphism, then locally $\Lambda$ may be described as the graph of some function. Of course, in general $\pi \mid \Lambda$ will not be a diffeomorphism, but will have certain singularities. For example, a

AMS(MOS) subject classifications (1970). Primary 35C10; Secondary 53B99.

Key words and phrases. Asymptotic solutions, singularity theory, Lagrangian manifold.

${ }^{1}$ Both authors were supported under NSF contract GP 22927. 
Lagrangian manifold may be formed by pasting together along a caustic the graphs of two solutions of the eikonal equation in a given domain. This pasting is smooth in $T^{*}(X)$; only in the base do singularities appear.

If $\Lambda$ is a Lagrangian manifold, we call a point $\left(x_{0}, \xi_{0}\right) \in \Lambda$ regular if the Jacobian of the projection $\pi: \Lambda \rightarrow X$ has rank $n$ at $\left(x_{0}, \xi_{0}\right)$. Given a regular point $\left(x_{0}, \xi_{0}\right), \pi$ restricted to a sufficiently small neighborhood is a diffeomorphism; hence near a regular point $\Lambda$ may be parametrized as the graph of a function, often called a phase function. The following proposition, proved in Chapter 3, $\S 1$ of [3], shows that in a certain sense one can parametrize a Lagrangian manifold by a phase function even at singular points.

Proposition. Suppose $d \pi$ has rank $n-i$ at $\left(x_{0}, \xi_{0}\right)$. Then there exists a function $\phi=\phi(x, \theta)$ on $X \times R^{i}$ such that its critical set $C_{\theta}=\{(x, \theta)$ : $\left.d_{\theta} \phi=0\right\}$ is an $n$-dimensional submanifold of $X \times R^{i}$ and the map $C \rightarrow T_{X}^{*}$, $(x, \theta) \rightarrow\left(x, d_{x} \phi\right)$ maps a neighborhood of $\left(x_{0}, 0\right)$ diffeomorphically onto a neighborhood of $\left(x_{0}, \xi_{0}\right)$ in $\Lambda$.

We shall call the set $S \subset \Lambda$ of singular points the Maslov cycle and shall call $\pi(S)$ the caustic of $\Lambda$. We may decompose $S$ as a disjoint union

$$
S=S_{1} \cup S_{2} \cup \cdots \cup S_{n},
$$

$S_{j}$ consisting of points where $d \pi$ has rank exactly $n-j$. In [2] we have shown that for $\Lambda$ in general position $S_{j}$ is a submanifold of $\Lambda$ of codimension $j(j+1) / 2$. If $\Lambda$ is in general position we shall call a point $\left(x_{0}, \xi_{0}\right)$ of $S_{1}$ a fold point if the kernel of $d \pi$ at $\left(x_{0}, \xi_{0}\right)$ and the tangent space of $S_{1}$ together span $T(\Lambda)$. For example the Lagrangian manifold constructed above by pasting along a caustic has fold points above the caustic, provided that the caustic is a smooth convex curve (the principal case considered by Ludwig).

Now for each $i, i=0, \ldots, n$, let $P_{i}(x, D)$ be a partial differential operator on $X$ of order $i$ and with real leading symbol, $P_{i}(x, \xi)$. Consider the partial differential operator

$$
P(x, D, \lambda)=\sum_{i=0}^{m}\left(\frac{1}{\sqrt{ }-1 \lambda}\right)^{i} P_{i}(x, D)
$$

with $\lambda$ a large parameter. We will call the sum $\sum P_{i}(x, \xi)$ the symbol of this operator. If this symbol vanishes identically on $\Lambda$ then the Maslov theory produces global asymptotic solutions of $(\mathrm{C})$ by patching together local solutions which are of the form

$$
\int a(x, \theta, \lambda) e^{i \lambda \phi(x, \theta)} d \theta,
$$


where $\phi$ is a phase function parameterizing $\Lambda$ and $a(x, \theta, \lambda)$ is an asymptotic series in powers of $\lambda^{-1}$. At a regular point, no $\theta$ variables are required in (D), and the expression reduces to a form analogous to (B). The following theorem, which contains Ludwig's result as a special case, shows that at a fold point (D) may again be simplified.

THEOREM. Let $\left(x_{0}, \xi_{0}\right)$ be a fold point on the Maslov cycle. Then there exist smooth functions $\rho_{0}$ and $\rho_{1}$ near $x_{0}$ such that $(\mathrm{D})$ is asymptotic to

$$
e^{i \lambda \rho_{0}}\left\{\frac{a_{0}(x, \lambda)}{\lambda^{1 / 3}} \operatorname{Ai}\left(\lambda^{2 / 3} \rho_{1}\right)+\frac{a_{1}(x, \lambda)}{\lambda^{2 / 3}}(\operatorname{Ai})^{\prime}\left(\lambda^{2 / 3} \rho_{1}\right)\right\}
$$

where $a_{i}(x, \lambda)$ is an asymptotic series in $\lambda^{-1}$. Moreover $\left(d \rho_{1}\right)_{x_{0}} \neq 0$, and the caustic is locally defined by the equation $\rho_{1}=0$.

(We note, in passing, that this formula also appears in the classical WKB solutions of the reduced Schrödinger equation near a turning point; this appearance is also due to a fold singularity in the associated Lagrangian manifold.)

We only sketch the proof of this theorem. The first step is a canonical form for phase functions which give a fold point.

Proposition. If $\left(x_{0}, \xi_{0}\right)$ is a fold point then one can parametrize $\Lambda$ near $\left(x_{0}, \xi_{0}\right)$ by a phase function $\phi=\phi(x, \theta)$ on $X \times R$ of the form

$$
\phi(x, \theta)=\rho_{0}(x)+\rho_{1}(x) \theta+\theta^{3} / 3
$$

where $\left(d \rho_{1}\right)_{x_{0}} \neq 0$. The caustic is locally defined by the equation $\rho_{1}=0$.

(One proof of this uses a $C^{\infty}$ variant of an argument of Chester-Friedmann-Ursell [1] and another uses the Malgrange preparation theorem. Both proofs can be found in [2].)

Now let $a(x, \theta)$ be a smooth function of $x$ and $\theta$ and let $\phi$ be the function (F). By the Malgrange preparation theorem we can write

$$
a(x, \theta)=a_{0}(x)+a_{1}(x) \theta+h(x, \theta) \partial \phi / \partial \theta
$$

in a neighborhood of $\left(x_{0}, 0\right)$. Therefore, given an integral of the form

$$
\int a(x, \theta) e^{i \lambda \phi(x, \theta)} d \theta
$$

we can by an integration by parts write it in the form

$$
\int\left(a_{0}(x)+a_{1}(x) \theta-\frac{1}{\lambda} \frac{\partial h(x, \theta)}{\partial \theta}\right) e^{i \lambda \phi(x, \theta)} d \theta
$$

By repeated application of this argument we see that the expression (D) can be rewritten in the simpler form 


$$
a_{0}(x, \lambda) \int e^{i \lambda \phi(x, \theta)} d \theta+a_{1}(x, \lambda) \int \theta e^{i \lambda \phi(x, \theta)} d \theta
$$

where $a_{0}(x, \lambda)$ and $a_{1}(x, \lambda)$ are asymptotic series in $\lambda^{-1}$.

But we recall that the Airy function $\mathrm{Ai}(t)$ has an integral representation of the form

$$
\operatorname{Ai}(t)=\frac{1}{2 \pi} \int_{-\infty}^{\infty} e^{i\left[t \theta+\theta^{3} / 3\right]} d \theta .
$$

Substitution of this expression into $(\mathrm{G})$ yields $(\mathrm{C})$.

In [2] we present formulas analagous to (E) for some more complex singularities of $\Lambda$. In particular, simplification of (D) at points where $\Lambda$ has one of the Moran singularities $\left(S_{1,1}, S_{1,1,1}\right.$, etc., in the terminology of [7]) leads to the "generalized Airy functions" defined by Ludwig in $\$ 4$ of his paper. The case of intersecting focal surfaces, not treated by Ludwig, corresponds to a $S_{2}$ singularity of $\Lambda$. Generically, this occurs for the first time in 3 dimensions and there are two distinct stable cases, called hyperbolic and elliptic in [8]. The associated generalized Airy functions are

and

$$
A(x, y, z)=\iint \exp \left(i\left(x \alpha+y \beta+z \alpha \beta+\frac{\alpha^{3}+\beta^{3}}{3}\right)\right) d \alpha d \beta
$$

$$
B(x, y, z)=\iint \exp \left(i\left(x \alpha+y \beta+z\left(\alpha^{2}+\beta^{2}\right)+\frac{\alpha^{3}-3 \alpha \beta^{2}}{3}\right)\right) d \alpha d \beta
$$

respectively. The asymptotic solution (D) simplifies to a series involving the functions $A$ or $B$ and their first partial derivatives. These results are proved in [2], along with an analysis of the asymptotic thickness of the various transition layers and relative intensities which occur.

\section{BIBLIOGRAPHY}

1. C. Chester, B. Friedmann and F. Ursell, An extension of the method of steepest descent, Proc. Cambridge Philos. Soc. 54 (1957), 599-611. MR 19, 853.

2. V. Guillemin and D. Schaeffer, Maslov theory and singularities, M.I.T., Cambridge, Mass., 1972. (Mimeographed notes.)

3. L. Hörmander, Fourier integral operators. I, Acta Math. 127 (1971), 79-183.

4. D. Ludwig, Uniform asymptotic expansions at a caustic, Comm. Pure Appl. Math. 19 (1966), 215-250. MR 33 \# 4446.

5. V. Maslov, Perturbation theory and asymptotic methods, Moskov. Gos. Univ., Moscow, 1965. (Russian)

6. - Characteristics of pseudo-differential operators, Proc. Internat. Congress Math. (Nice, 1970), vol. 2, Gauthier-Villars, Paris, 1971, pp. 755-769.

7. R. Thom and H. Levine, Singularities of differentiable mappings, Proc. Liverpool Singularities Sympos. I (1969/70), Lecture Notes in Math., vol. 192, Springer-Verlag, Berlin and New York, 1971, pp. 1-89.

8. R. Thom, Morphogenesis, Addison-Wesley, Reading, Mass., 1972.

Department of Mathematics, Massachusetts Institute of Technology, Cambridge, MASSACHUSETTS 02139. 